\title{
Analysis of interval histograms of period of the electroencephalogram from homologous left and right derivations in verbal and nonverbal tasks
}

\author{
WALTER W. SURWILLO \\ Department of Psychiatry \& Behavioral Science, University of Louisville School of Medicine, \\ Louisville, Kentucky 40201
}

\begin{abstract}
Interval histograms of period of the electroencephalogram (EEG) were derived from parietaloccipital derivations of left and right hemispheres in 10 right-handed males, aged 5-11 years. The EEGs were obtained during performance of (1) a verbal task in which the subject was asked to listen to and recall, in correct order, lists of digits equal in length to his digit span, (2) a nonverbal task in which the subject pushed a button as quickly as possible whenever a 1,000- $\mathrm{Hz}, 72-\mathrm{dB}$ tone was heard over a loudspeaker. The histograms, which were derived by computer, were composed of the durations of $380 \mathrm{EEG}$ half waves taken immediately prior to the initiation of the stimuli. The histograms from left and right derivations, for each condition, were characterized in terms of their central tendency $(m)$, dispersion $(\sigma)$, skewness $\left(\beta_{1}\right)$, and kurtosis $\left(\beta_{2}\right)$. Except for the values of $m$ in the case of the nonverbal task, there were no differences between the parameters obtained from left and right hemispheres. The fact that $\sigma, \beta_{1}$, and $\beta_{2}$ showed no interhemispheric differences argued strongly in favor of the reliability of the central-moments technique of EEG period analysis. The values of $\mathrm{m}$ from left and right hemispheres were more alike during performance of the verbal than of the nonverbal task. These findings suggest that performance of verbal and nonverbal tasks may be accompanied by differences in left-right symmetry of the EEG and that the central-moments technique may be sensitive to these interhemispheric differences.
\end{abstract}

In a recent article, Surwillo (1975) reported a technique for analyzing the electroencephalogram (EEG) which appeared to be useful in investigating the brain electrical changes that take place during growth and development. The technique involves casting measurements of the duration of a large number of half wavelengths in the EEG into an interval histogram and then characterizing the resulting distribution in terms of its first four central moments. Surwillo (1975) found that the first four central moments - which are measures of the central tendency, dispersion, skewness, and kurtosis of a distribution-proved to be useful predictors of children's ages between 5 and 17 years.

One important question relating to the centralmoments technique of EEG period analysis concerns its reliability. We know that EEGs recorded simultaneously from bilaterally homologous areas of the two hemispheres in the same subject show a high degree of synchrony (Adrian \& Yamagiwa, 1935; Brazier \& Casby, 1952; Cohn, 1948; Cooper \& Mundy-Castle, 1960; Garoutte \& Aird, 1958; Liske, Hughes, \& Stowe, 1967). This being the case, we should expect the central moments of histograms

This research was supported by PHS Research Grant HD-02395 from the National Institute of Health. derived from homologous areas of the two hemispheres to show close similarities. One purpose of the present investigation was to bring some evidence to bear on this question.

Another facet of the present study relates to recent work which suggests that EEGs from homologous areas of the two hemispheres show differences according to whether a task performed at the time the EEGs are taken involves verbal or nonverbal activities (Callaway \& Harris, 1974; Doyle, Ornstein, \& Galin, 1974; Galin \& Ornstein, 1972; Morgan, Macdonald, \& Hilgard, 1974). An obvious question relevant to these findings concerns whether the central-moments technique of EEG period analysis might be sensitive to hemispheric differences associated with cognitive mode of a task. The second objective of the present investigation addressed this question; we sought to determine if the first four central moments of interval histograms from bilateral EEGs differ in relation to whether a verbal or a nonverbal task is being performed.

\section{METHOD}

\section{Subjects}

The experimental sample consisted of 10 healthy, right-handed boys between the ages of 5 and 11 years, and having a mean age of 8 years. All boys were volunteers who were attending local 
schoo:s and were performing at grade level or better as judged by their teachers. The subjects were selected from a larger group of boys brought to the laboratory by their parents to have their EEGs recorded. Prior to taking any data, certificates of informed consent were signed by the parents. Selection criteria for the experiment required that the EEGs be generally free of contamination by extracerebral artifacts-principally electromyograms, electrocardiograms, and electrical activity associated with eye and eyelid movements. Persons whose EEGs showed any evidences of electrocardiograms or tonic muscle tension levels $\geqslant 5 \mu \mathrm{V}$ were rejected outright from the study. Anyone unable to keep his eyes and eyelids still during the test procedure was likewise excluded.

\section{Procedure}

Testing was carried out in a dimly lighted, electrostatically shielded and sound-insulated room while the subject rested in a semirecumbent position with his eyes closed. Each subject participated in two different activities-a nonverbal task and a verbal task. EEGs were recorded while the tasks were actually being performed.

In the nonverbal activity, the subject performed a simple reaction-time (RT) task in which the stimulus was a $72-\mathrm{dB}$, $1,000-\mathrm{Hz}$ tone that was presented over a loudspeaker. Procedural details were as reported in an earlier study (Surwillo, 1971). Briefly, the subject held a response key in his right hand and pressed it whenever the $1,000-\mathrm{Hz}$ tone occurred. Twenty-one such tones were presented at random and without warning during an 8-min test session. Two test sessions consisting of 21 trials each were given after the subject had received sufficient practice to assure that he understood the task and was pressing the response key as quickly as possible. The $1,000-\mathrm{Hz}$ tones were triggered by a technician who ran the EEG recording equipment which was located in an adjacent room. The technician presented the tones according to a prearranged schedule using a $1 / \mathrm{sec}$ marker pen on the EEG machine to time the intervals between consecutive stimuli.

The verbal task involved listening to and recalling aloud lists of digits that were played to the subject over a loudspeaker. Twentyone different lists of digits were presented in the same manner as the 21 tones in the nonverbal task. Each list consisted of digits that were drawn at random from the numbers 1-9. The number of digits in a list was equal to the subject's digit span, which was determined prior to the start of this task by means of the WISC Digit Span Forward Test. Thus, for example, a subject whose digit span was five digits on the WISC test was played the prerecorded tape consisting of 21 five-digit numbers. In all cases, digits were presented at the standard rate of $1 / \mathrm{sec}$, and the subject was required to recall the digits in the same order immediately after the last digit of a list was heard.

\section{Recording and Quantification of EEG}

EEGs were recorded on a Beckman Type $\mathbf{R}$ dynograph which had a bandwidth of 1.6-32 $\mathrm{Hz}$ (response down $3 \mathrm{~dB}$ at the cutoff frequencies). Four sponge-type surface electrodes were placed on the scalp over $\mathrm{O}_{1}, \mathrm{O}_{2}, \mathrm{P}_{3}$ and $\mathrm{P}_{4}$ in the "10-20" International System, and held in place by means of a headgear fashioned from strips of lastonet bandage. A ground electrode was attached to the dorsal surface of the neck, approximately over the sixth cervical vertebra. Four channels of EEGs corresponding to left and right parietal-occipital, transverse parietal, and transverse occipital derivations were traced out at a chart speed of $50 \mathrm{~mm} / \mathrm{sec}$ and a deflection sensitivity of 5 microvolts $/ \mathrm{mm}$. An Ampex FR-1300 Instrumentation Recorder was used to make parallel recordings of the data on analog tape for of fline analysis.

The EEG period analysis was done off-line on a PDP-8/L computer using the "look back" mode of operation of a program which was described earlier (Sharp, Smith, \& Surwillo, 1975). This program measured, to $1-\mathrm{msec}$ accuracy, the durations of a sample of half-waves taken from the intervals between the trials in the digit-recall task and from the intervals between the trials in the second session of the RT task. The measurements of half-wave duration $(1 / 2 D)$ for the verbal and nonverbal tasks were cast into separate distributions. These distributions, which had a class interval of $5 \mathrm{msec}$, were outputed on the chart of a teletypewriter as interval histograms.

Separate histograms were obtained for the parietal-occipital recordings from the left and right hemispheres of each subject. Each histogram for the verbal task was composed of 380 measurements of $1 / 2 \mathrm{D}$; the measurements were taken from the 19 consecutive half-waves immediately preceding the first digit in lists $2-21$ of the digit-recall task. For the RT task, the histograms also consisted of 380 measurements of $1 / 2 \mathrm{D}$. In this case, the 19 consecutive half-waves which came immediately before the stimulus in Trials 2-21 of Session 2 were measured.

Prior to running the data on the computer, the strip-chart recordings were inspected to insure that the portions of the EEGs selected for analysis were free of extracerebral artifacts. Tracings from the transverse derivations were used to help in the identification of artifacts-mainly electromyograms due to increased tension in muscles of the neck and electrical changes associated with eye and eyelid activity. The tracing from the occipital derivation was used to monitor muscle-tension artifacts. Whenever muscle tension recorded in this tracing exceeded $5 \mu \mathrm{V}$, data from the left and right derivations were not analyzed. Blinks and gross eye movements were readily detected by searching the tracing from the parietal derivation for the characteristic, easily recognizable waveforms. If a blink or eye movement was found in the parietal recording, data from the left and right derivations which fell in that interval were not analyzed. As the subjects in the study had been selected because their EEGs were mainly free of artifacts, there were few instances in which recordings intended for analysis were rejected because of contamination by such activity.

\section{Data Analysis}

For each subject, a histogram was obtained from left- and right-hemisphere recordings for the verbal and the nonverbal tasks. Each of these 40 interval histograms was examined to confirm that the distribution had but a single mode. Findings from an earlier study (Surwillo, 1975) suggested that such distributions were usually unimodal. Central tendency, dispersion, skewness, and kurtosis of the histograms were defined, respectively, by the mean $(\mathrm{m})$, standard deviation $(\sigma), \beta_{1}$, and $\beta_{2}$ of the distributions. $\beta_{1}$ and $\beta_{2}$ are based, respectively, on the ratio of the third and fourth central moments of a distribution to the distribution's standard deviation. Unlike the third and fourth moments, $\beta_{1} s$ and $\beta_{2}$ s of two distributions may be compared directly as they take the dispersion-which affects the value of the higher-order moments (Kendall \& Stuart, 1963; Stilson, 1966)-into account.

\section{RESULTS}

Table 1 shows group averages of $m, \sigma, \beta_{1}$, and $\beta_{2}$ of the distributions from left and right derivations for the RT and the digit-recall tasks. It is clear that the distributions of $1 / 2 \mathrm{D}$ from left and right derivations were closely similar for both tasks. Except for the values of $m$ in the nonverbal task which differed significantly at the .01 level of confidence, none of the distribution parameters from left and right derivations differed significantly.

Figure 1 shows selected samples of the raw EEGs recorded in the interval immediately preceding the 
Table 1

Average Values $(\mathrm{N}=10)$ of the Statistics $\mathrm{m}, \sigma, \beta_{1}$, and $\beta_{2}$ of the Distributions of $1 / 2 \mathrm{D}$ from Left and Right Parietal-Occipital Derivations for Verbal and Nonverbal Tasks*

\begin{tabular}{lccccc}
\hline & \multicolumn{2}{c}{ Verbal Task } & & \multicolumn{2}{c}{ Nonverbal Task } \\
\cline { 2 - 3 } \cline { 6 - 7 } \multicolumn{1}{c}{ Statistic } & left & right & & left & right \\
\hline m (milliseconds) & 54.9 & 55.4 & & 52.2 & 54.7 \\
$\sigma$ (milliseconds) & 22.8 & 22.2 & & 23.2 & 23.0 \\
$\beta_{1}$ & 1.44 & 1.87 & & 1.51 & 1.77 \\
$\beta_{2}$ & 6.21 & 7.56 & & 5.76 & 6.51 \\
\hline
\end{tabular}

*For each distribution of $1 / 2 D, n=380$.

first digit in the verbal task. The tracings are from 8 of the 20 trials in subjects 1 and 3 . To illustrate some of the properties of the distribution parameters in relation to the raw tracings, we computed $\mathrm{m}, \sigma, \beta_{1}$, and $\beta_{2}$ of the interval histograms based on a total of $1521 / 2$ Ds taken from these data. Although the small $\mathrm{n}$ severely limits the reliability of the estimates of $\beta_{1}$ and $\beta_{2}$, the values of $m$ and $\sigma$ (see legend of Figure 1) reflect what is apparent from inspection of the raw tracings. Note in Figure 1 that, on the whole, the durations of the $1 / 2$ waves in subject 3 are longer and more variable than they are in subject 1 . Also, the tracings from left and right hemispheres look to be more similar in subject 1 than in subject 3 . These observations are readily corroborated by examination of the ms and os of the interval histograms. Thus, ms and os for subject 3 are larger than for subject 1 . Similarly, ms and $\sigma$ s from left and right hemispheres are more similar in subject 1 than in subject 3.

\section{DISCUSSION}

The close similarity of the measures of central tendency, dispersion, skewness, and kurtosis of the distributions from left and right hemispheres of the same subjects during performance of the verbal task argues strongly in favor of the reliability of the central-moments technique. As the accuracy with which third and fourth moments can be computed varies profoundly with sample size (Elderton \& Johnson, 1969), greater correspondence between $\beta_{1} s$ and $\beta_{2}$ s could undoubtedly have been achieved by increasing the $\mathrm{n}$ of the distributions. Nevertheless, the absence of any significant differences between $\mathrm{m}$, $\sigma, \beta_{1}$, and $\beta_{2}$ for the verbal task, and between $\sigma, \beta_{1}$, and $\beta_{2}$ for the nonverbal task, suggests that an $n$ of 380 is sufficiently large for practical purposes.

The statistically significant difference of $2.5 \mathrm{msec}$ between the means of the distributions for the nonverbal task amounts to an average difference in EEG period of $5 \mathrm{msec}$ between the hemispheres. This value is equivalent in terms of frequency to about $.4 \mathrm{~Hz}$. The results appear to be due to a shift in frequency of the dominant rhythm of the left and/or right hemisphere rather than the differential contribution to the mean of high or low frequency activity from either hemisphere. Evidence in support of this conclusion derives from the fact that the raw data reveal actual shifts in the peak of the distribution from one hemisphere relative to the peak of the distribution from the other, and from the finding that the average values of dispersion, skewness, and kurtosis of the left-hemisphere distributions (Table 1) were no different from the righthemisphere values. This argument would seem to effectively rule out the possibility that the left-right difference observed in the nonverbal task was due to the presence of greater amounts of muscle-potential artifacts in the left-hemisphere recordings.

The present findings suggest that period of the

\section{SUBJECT \#।}

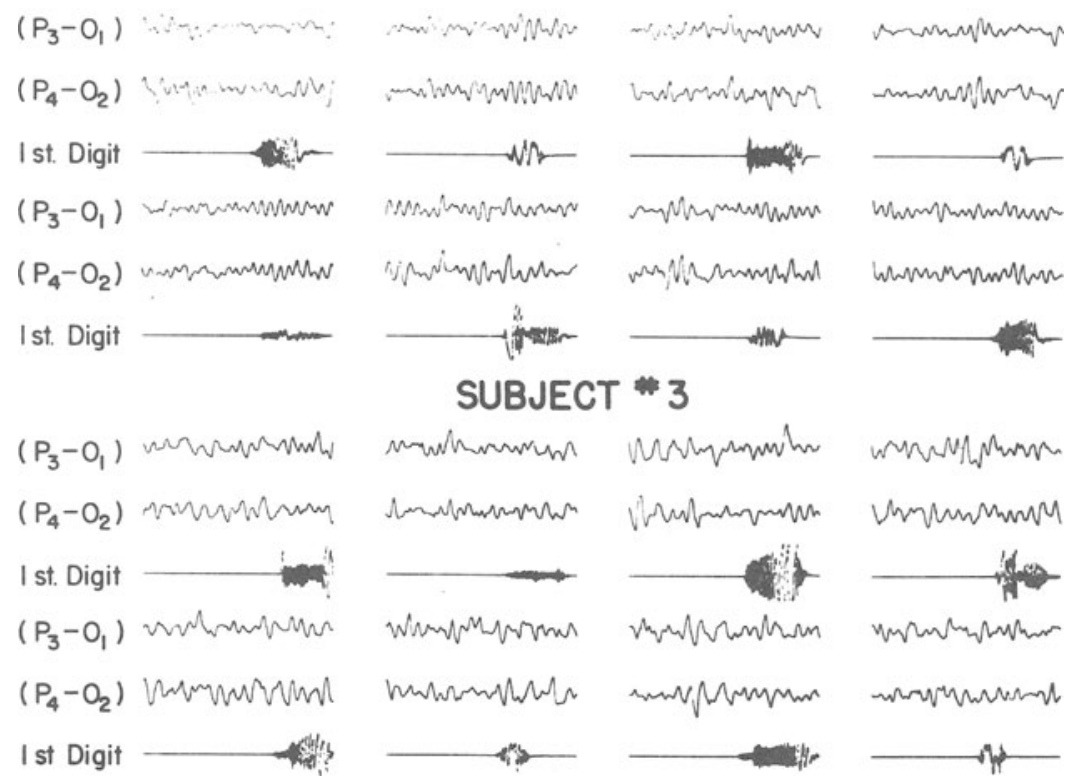

Figure 1. Selected samples of raw EEGs recorded from left $\left(\mathrm{P}_{3}-\mathrm{O}_{1}\right)$ and right $\left(\mathrm{P}_{4}-\mathrm{O}_{2}\right)$ hemispheres in subjects 1 and 3 . Tracings show waves in the interval immediately preceding the first digit in 8 of the 20 trials of the verbal task. Analysis of histograms composed of 19 half-waves taken from each of these trials (total $1 / 2 D=152$ ) yielded the following parameters. In each case, the first number listed is the value for the left hemisphere while the second is for the right. Subject 1: $m=54.3,55.9 ; \sigma=18.8$, 19.9; $\beta_{1}=.49, .96 ; \beta_{2}=5.68,6.82$. Subject $3: m=60.8,62.9 ; \sigma=34.0,25.1 ; \beta_{1}=1.49$, $.67 ; \beta_{2}=5.28,4.96$. Note that the differences in the values of $m$ and $\sigma$ between subjects and between hemispheres are clearly apparent in a visual inspection of the raw tracings. See text. 
EEG from homologous areas of the two hemispheres may be more alike during performance of verbal than of nonverbal tasks. While EEG period can hardly be taken as a measure of synchrony between the hemispheres, our results are indicative of increased interhemispheric symmetry during performance of the verbal task. In this sense, perfect symmetry would occur when distributions of $1 / 2 D$ from left and right hemispheres were identical, while deviations from symmetry would occur as the parameters of the distributions became more dissimilar. Thus, the present report suggests that tasks utilizing different cognitive modes of performance may result in differences in left-right symmetry, and that the central-moments technique of EEG period analysis may be sensitive to these interhemispheric differences.

It should be borne in mind, of course, that the data analyzed in this experiment were from EEGs recorded immediately prior to the presentation of the stimulus objects. In so doing, we assumed that the appropriate cognitive strategy employed by the subject in the analysis of the stimulus object is present throughout the entire session and not just during the time that the stimulus occurs and the response is made. This assumption seemed justified not only by the simplicity of the resulting experimental design, but by the general impression that the cognitive aspects of performing the two tasks were indeed different throughout the entire sessions and not just when stimuli occurred and responses were made. Nevertheless, we cannot say how the findings might differ had the data analyzed come from EEGs recorded in the intervals between presentation of the stimulus object and the initiation of verbal report or motor response. This matter is an appropriate question for further investigation.

The presence of interhemispheric EEG differences in relation to cognitive mode of the task performed is hardly surprising in the light of present knowledge concerning brain functioning. There is some evidence from studies of brain-injured and neurosurgical patients which indicates that the two hemispheres of the brain are not alike in regard to function (Gazzaniga, 1970; Geschwind, 1970; Luria, 1966; Milner, 1967; Sperry, Gazzaniga, \& Bogen, 1969). This evidence suggests that the left hemisphere mediates verbal activities and mathematical calculation but is severely limited in dealing with nonverbal tasks and problems involving spatial relationships. By contrast, the right hemisphere displays very little verbal functioning or facility in calculation, but can perform a variety of nonverbal tasks. Whether the central-moments technique of analysis can be useful in identifying degree of hemispheric involvement in the performance of a particular task, however, is a question which awaits future investigation.

\section{REFERENCES}

Adrian, E. D., \& Yamagiwa, K. The origin of the Berger rhythm. Brain, 1935, 58, 323-351.

Brazier, M. A. B., \& Casby, J. U. Crosscorrelation and autocorrelation studies of electroencephalographic potentials. Electroencephalography and Clinical Neurophysiology, 1952. 4. 201-211.

Callaway, E., \& Harris, P. R. Coupling between cortical potentials from different areas. Science, 1974, 183, 873-875.

CонN, R. The occipital alpha rhythm: A study of phase variations. Journal of Neurophysiology, 1948, 11, 31-37.

Cooper, R., \& Mundy-Castle, A. C. Spatial and temporal characteristics of the alpha rhythm: A toposcopic analysis. Electroencephalography and Clinical Neurophysiology, 1960, 12. 153-165.

Doyle, J. C., Ornstein, R., \& Galin, D. Lateral specialization of cognitive mode: II. EEG frequency analysis. Psycho phvsiology, 1974, 11, 567-578.

Elderton, W. P., \& Johnson, N. L. Systems of frequency curves. Cambridge: Cambridge University Press, 1969.

Galin, D., \& Ornstein, R. Lateral specialization of cognitive mode: An EEG study. Psychophysiology, 1972, 9, 412-418.

Garoutte, B., \& AIRD, R. B. Studies on the cortical pacemaker: Synchrony and asynchrony of bilaterally recorded alpha and beta activity. Electroencephalography and Clinical Neurophysiology, 1958, 10, 259-268.

Gazzanica, M. S. The bisected brain. New York: Appleton(entury-(rofts, 1970).

Geschwind, N. The organization of language and the brain. Science, 1970, 170, 94().944.

Kendal.., M. G., \& Stuart, A. The advanced theory of statistics (Vol. 1). New York: Hafner, 1963.

Liske, E., Hughes, H. M., \& Stowe, D. E. Cross-correlation of human alpha activity: Normative data. Electroencephalography and Clinical Neurophysiology, 1967, 22, 429-436.

LURIA, A. R. Higher cortical functions in man. New York: Basic Books, 1966.

MilneR, B. Brain mechanisms suggested by studies of temporal lobes. In C. H. Millikan \& F. L. Darley (Eds.), Brain mechanisms underlying speech and language. New York: Grune \& Stratton, 1967. Pp. 122-145.

Morgan, A. H., Macdonald, H., \& Hilgard, E. R. EEG alpha: Lateral asymmetry related to task, and hypnotizability. Psychophysiology, 1974, 11, 275-282.

Sharp, F. H., Smith, G. W., \& Surwillo, W. W. Period analysis of the electroencephalogram with recording of interval histograms of EEG half-wave durations. Psychophysiology, 1975, 12, 471-475.

Sperry, R. W., Gazzaniga, M. S., \& Bogen, J. E. Interhemispheric relationships; the neocortical commissures; syndromes of hemispheric disconnection. In P. S. Vilden \& G. W. Bryn (Eds.), Handbook of clinical neurology (Vol. 4). Amsterdam: North Holland, 1969. Pp. 273-290.

Stilson, D. W. Probability and statistics in psychological research and theory. San Francisco: Holden-Day, 1966.

Surwillo, W. W. Human reaction time and period of the EEG in relation to development. Psychophysiology, 1971, 8, 468-482.

SuRwillo, W. W. Interval histogram analysis of period of the electroencephalogram in relation to age during growth and development in normal children. Psychophysiology, 1975, 12, 506-512.
(Received for publication November 26, 1975; revision received February 23, 1976.) 